\title{
Use of the esophageal echo-Doppler to guide intensive care unit resuscitations: A retrospective study
}

\author{
Peter S. Stawicki, William S. Hoff*, James Cipolla*, Nathaniel McQuay Jr, Michael D. Grossman*
}

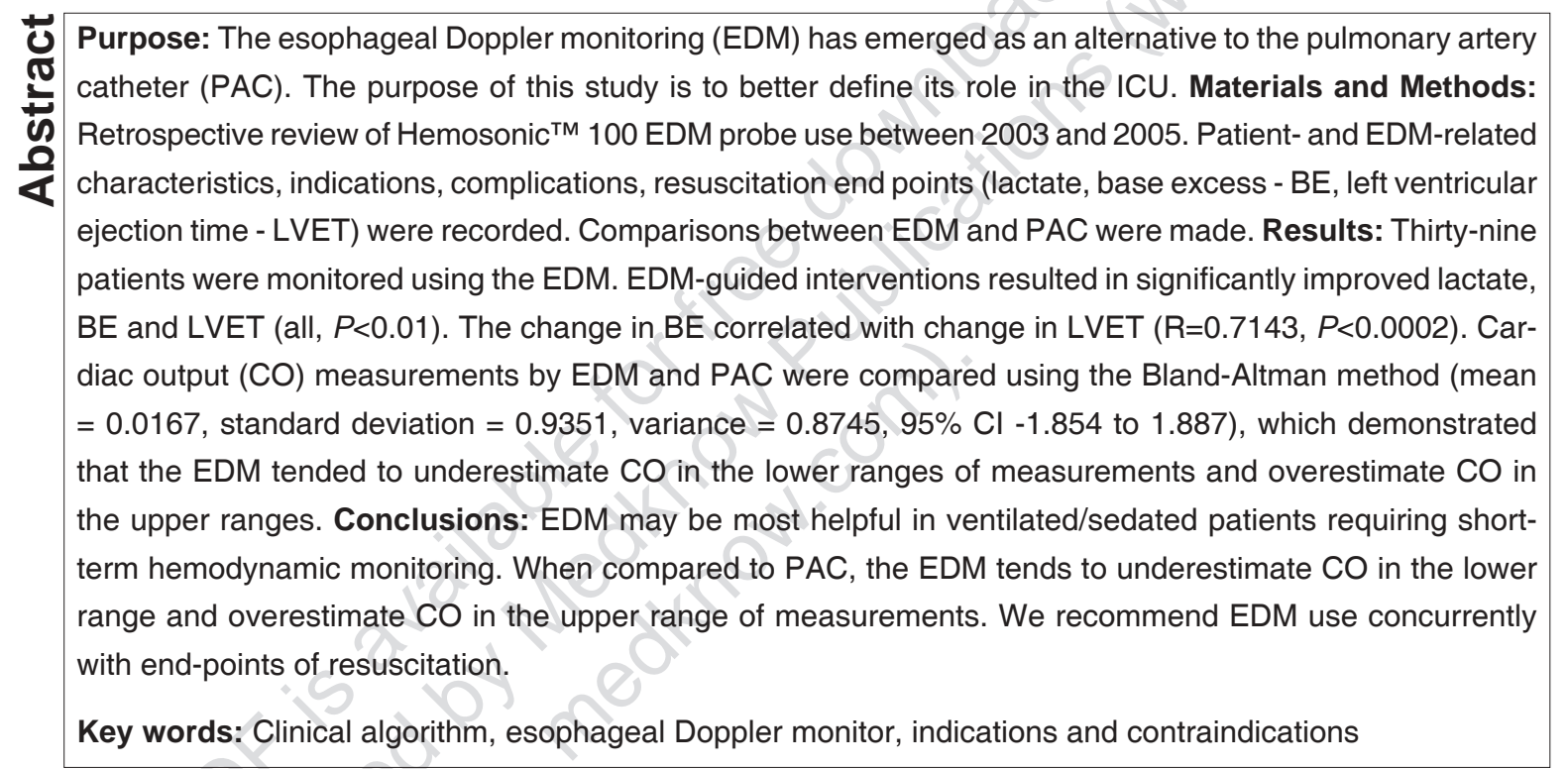

\section{Introduction}

The pulmonary artery catheter (PAC) is considered the standard of care in invasive hemodynamic management of critically ill intensive care unit (ICU) patients. ${ }^{[1]}$ Despite its potential risks, controversies and requirement for specialized critical care environment, the PAC continues to be widely utilized. ${ }^{[2,3]}$ The esophageal

\section{From:}

Stawicki Research Foundation, King of Prussia PA, *St Luke's Regional Level I Resource Trauma Center, Bethlehem PA and University of

Pennsylvania Trauma Network, Philadelphia PA, USA

\section{Correspondence to:}

Dr. S. Peter Stawicki, 304 Monroe Blvd, King of Prussia, PA 19406, USA.

E-mail: stawicki_ace@yahoo.com
Doppler monitoring (EDM) technology has emerged as a potential alternative to the PAC. ${ }^{[4-6]}$ Numerous studies have established the safety and reliability record of the EDM in various clinical settings. ${ }^{[6-10]}$ The purpose of this study is to analyze our institutional use of the EDM and design an algorithm to define the role for the EDM in our intensive care units.

\section{Materials and Methods}

A retrospective review of EDM use from January 2003 to September 2005 was performed. The Hemosonic ${ }^{\mathrm{TM}}$ 100 (Arrow International, Inc., Reading, Pennsylvania, USA) esophageal echo Doppler probe was utilized. 
Patient characteristics, indications for EDM use, insertion time and route, duration of use, complications and resuscitation outcomes were recorded. Measured parameter comparisons (cardiac output) between the EDM and the PAC were performed.

The Hemosonic ${ }^{\mathrm{TM}} 100$ EDMonitor consists of a reusable trans-esophageal probe with sterile, single-use jackets. The probe utilizes two ultrasonic transducers interposed on the tip of the probe: A continuous M-mode transducer operates at $10 \mathrm{MHz}$ and the pulsed Doppler transducer operates at $5 \mathrm{MHz}$. The probe can be inserted orally or nasally. The probe is in the correct anatomical position when the Doppler stethoscope and waveform are at their maximal intensity, triphasic, with minimal distortion and the M-mode echo shows the parallel anterior and posterior walls of the descending aorta with minimal artifact. $^{[11]}$

Difficult probe insertion was defined as insertion time greater than seven minutes (duration greater than the $75^{\text {th }}$ percentile of all measured insertion times) or instances where the EDM had to be removed and replaced due to inability or difficulty in placement or patient factors (i.e., morbid obesity, inability to pass the probe nasally, patient intolerance requiring sedation and presence of a complication).

The EDM-specific parameter, left ventricular ejection time (LVET), was utilized to assess and follow cardiac preload and outflow impedance. The LVET is the time difference between opening and closing of the aortic valve. Increases in preload and/or decreases in afterload will increase LVET. Conversely, LVET will decrease with decreases in preload and/or increases in afterload. ${ }^{[12]}$

Outcome was reported as observed 28-day mortality as well as mortality predicted by the SAPS II score. ${ }^{[13]}$ For the purpose of mortality description in this study, only nonorgan donors (NOD, 32 patients) were considered, with the organ donors (OD, seven patients) excluded. The SAPS II score was calculated for each NOD.

Therapeutic utility of the EDM was determined based on the success of the resuscitation. If lactate and base excess worsened following the resuscitation, the resuscitation was determined to be unsuccessful, even in the context of improving EDM parameters.
Statistical methods used included Student's T-test, descriptive statistics and calculations of variable correlation. The Bland-Altman method for two different techniques of measurement was utilized when examining the correlation between PAC and EDM. Statistical significance was set at alpha $<0.01$.

\section{Results}

A total of 39 pts (26 men, 13 women, mean age $63.4 \pm 17.2 \mathrm{y} / \mathrm{o}$ ) were monitored using the EDM [Table 1]. The most common indications for EDM use included postoperative resuscitation (17/39), resuscitation of non-surgical patients (13/39), diagnosing the type of hemodynamic dysfunction (11/39) organ donor optimization (7/39), inability to utilize PAC due to anatomically difficult central venous access (5/39), verification of PAC data (4/39), trauma resuscitation (2/39) and intraoperative monitoring (1/39). More than one indication per patient could be present (i.e., a patient with suspected cardiogenic shock who is undergoing postoperative resuscitation or a patient in septic shock with anatomically difficult central venous access).

For the purpose of mortality analysis, the seven organ donors (OD) were excluded. The mean SAPS score for the remaining 32 patients was $18.4 \pm 4.58$ (predicted mortality $31.8 \pm 13.7 \%$ ). The observed 28 -day patient mortality in this group of patients was $7 / 32$ (21.9\%).

Probe data provided therapeutically useful information in 36 pts (92.3\%). Significant decrease in serum lactic acid and base excess was observed following episodes of EDM-guided resuscitation. EDM parameters guided appropriate use of vasopressors in 21 patients (53.8\%) and/or fluid resuscitation/diuresis in 36 patients (92.3\%), which was reflected by the changes in mean serum lactate $(3.53 \pm 2.28$ vs $1.88 \pm 1.23, P<0.0030)$, base excess $(-6.99 \pm 4.41$ vs $-2.15 \pm 4.41, P<0.0002)$, as well as LVET (263 \pm 93.6 vs $343 \pm 70.3, P<0.0002)$. The change in base excess significantly correlated with the change in LVET $(\mathrm{R}=0.7143, P<0.0002)$ [Table 2].

The EDM and the PAC-derived cardiac output (CO) data were subjected to the Bland-Altman analysis, a method for comparing different measuring instruments, with observed mean of 0.0167 , standard deviation of 0.9351 , variance of 0.8745 and $95 \%$ confidence interval -1.854 to 1.887 [Figure 1]. Further examination of the Bland- 


\section{Table 1: Summary of study results}

\begin{tabular}{lc}
\hline Variable(s) & Value \\
Patient and EDM use-related parameters & $63.4 \pm 17.2$ years \\
Mean age & 26 men, 13 women \\
Patient gender & 7 patients \\
Organ donors (OD) & 32 patients \\
Non-organ donors (NOD) & $18.4 \pm 4.58$ \\
Mean SAPS score (NOD) & \\
Mortality (NOD patients) & $31.8 \pm 13.7 \%$ \\
Predicted & $21.9 \%$ \\
Observed & $12(30.8 \%)$ \\
Oral insertion & $27(69.2 \%)$ \\
Nasal insertion & \\
Insertion time (mean) & $5.67 \pm 2.71$ minutes \\
Duration of use (mean) & $4.50 \pm 4.67$ hours
\end{tabular}

Resuscitation end-points Lactic acid

Preresuscitation (mean)

Postresuscitation (mean)

Base excess (BE)

Preresuscitation (mean)

Postresuscitation (mean)

Left-ventricular ejection time (LVET)

Preresuscitation (mean)

Postresuscitation (mean)
$P$-value (if applicable)

Comment

Predicted mortality based on SAPS score.

Oral route utilized in

$6 / 7(85.7 \%)$ of OD and $7 / 32(21.8 \%)$ of NOD

Prolonged insertion

time if $>7 \mathrm{~min}$

Prolonged EDM

usage if more than 6-8 hours

Esophageal Doppler monitoring (EDM)

\section{Table 2: Proposed contraindications for esophageal Doppler monitor use}

Recent esophageal surgery

Cranial, oral, or maxillofacial trauma

Esophageal varices

Known esophageal diverticulum (relative)

Patient inability to tolerate esophageal probe insertion

(Including requirement for prohibitive level of sedation)

Patient refusal

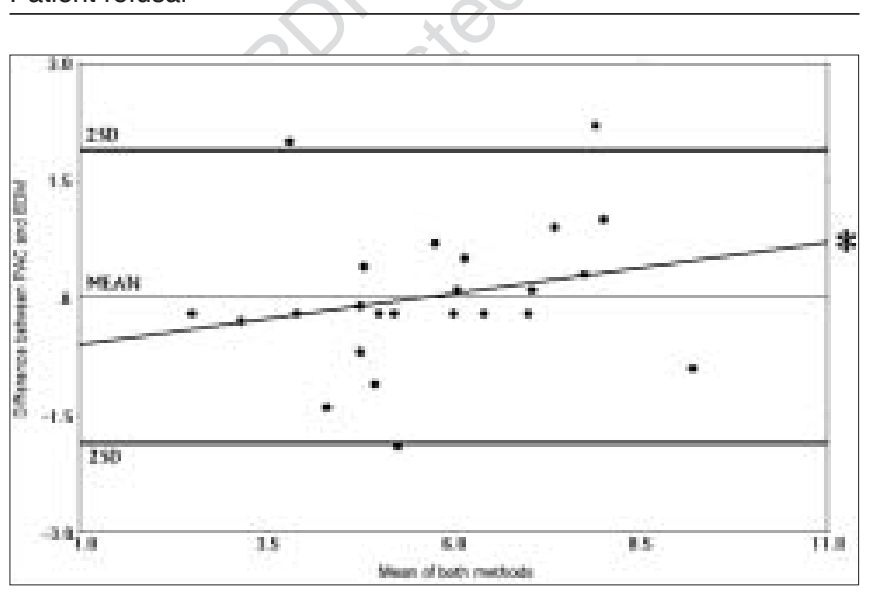

Figure 1: The Bland-Altman bias plot comparing cardiac output (CO) measurements obtained by the pulmonary artery catheter (PAC) and the esophageal Doppler monitor (EDM). The thin horizontal line indicates the mean (0.0167). Two thick horizontal lines indicate the $95 \%$ confidence interval $(-1.854$ to 1.887$)$. The trend line $\left({ }^{*}\right)$ demonstrates that the EDM tends to underestimate $\mathrm{CO}$ in the lower ranges of measurements and overestimate $\mathrm{CO}$ in the upper ranges relative to the PAC.
Altman bias plot demonstrated that the EDM tended to underestimate $\mathrm{CO}$ in the lower range of measurements and overestimate $\mathrm{CO}$ in the upper range.

The majority of patients met their resuscitation endpoints successfully within six hours $(75 \%)$ to eight hours (85\%) after initiating EDM-guided resuscitation. The three patients in whom EDM-guided resuscitation was not successful experienced clinical worsening and were documented to have increased lactate level and worsening base deficit despite resuscitative maneuvers.

The EDM was inserted orally (12/39) or nasally (27/39), depending on various patient factors and tolerability. Oral insertion was more likely in organ donors (6/7, 85.7\%) than non-organ donors (NOD) $(7 / 32,21.8 \%)$. Mean insertion time was $5.74 \pm 2.71 \mathrm{~min}$. Duration of probe use was $4.50 \pm 4.67 \mathrm{~h}$ (range $20 \mathrm{~min}$ to $19 \mathrm{~h}$, median $3.00 \mathrm{~h}$, first quartile 1.00 , third quartile $5.96 \mathrm{~h}$ ). Sedation was necessary in $28 / 39$ patients and $36 / 39$ patients were mechanically ventilated.

Difficult EDM insertion (insertion time $\geq 7$ min or $\geq$ 1 failed attempt at placement followed by successful placement) was encountered in $7 / 39(17.9 \%)$ patients. 
Factors related to difficult insertion included morbid obesity (4/39), epistaxis (1/39), vasovagal response $(1 / 39)$ and inability to insert nasally prompting conversion to oral insertion (1/39).

Complications included epistaxis in one patient and vasovagal response in another patient. The patient with epistaxis had narrow nasal opening and the EDM had to be placed orally. In the case of vasovagal response, the patient recovered from bradycardia promptly after removal of the probe without any interventions and subsequently underwent successful placement of the EDM.

There were no statistically significant correlations between parameters related to probe insertion (patient gender, age, weight/BMI, height, distance from probe tip to patient's nares or lips and prevalence of difficult insertion). However, we noted a trend towards difficult or prolonged probe insertion in morbidly obese patients and patients with small nares. We also noted more inter-measurement variability in the geriatric $(\geq 65$ year old) patients.

\section{Discussion}

The EDM has emerged as a reliable modality for noninvasive monitoring of critically ill patients in the ICU, the operating room and of critically ill obstetric patients. ${ }^{[4,8,14-15]}$ It offers useful hemodynamic data while (a) avoiding invasive line placement-related complications (pneumothorax, arterial injury, hematoma) and unnecessary needle exposure to the health care worker; (b) limiting the over-reliance on pulmonary arterial pressure-derived estimations of end-diastolic volume; and (c) obviating the technical limitations imposed by fluctuating intrathoracic pressures and relative lack of reliability of invasive arterial and central venous line monitoring, especially in the geriatric patient population. ${ }^{[4,6,16-17]}$

The most common indications for EDM use in this study included postoperative resuscitation, resuscitation of nonsurgical patients, diagnosis of the type of hemodynamic dysfunction, organ donor optimization, inability to utilize PAC due to anatomically difficult access, and verification of PAC data. Table 1 lists proposed absolute and relative contraindications to EDM placement.

The mean SAPS II score for nonorgan donor (NOD) group was $18.4 \pm 4.58$, with associated predicted 28 day mortality of $31.8 \pm 13.7 \%$. The observed 28 -day patient mortality was $21.9 \%$ in this study. Both the SAPS II-predicted and the observed mortality demonstrate the significant illness severity in this group of patients and support the use of either invasive or noninvasive hemodynamic monitoring tools.

We believe that the optimal role for the utilization of the EDM in the ICU is for initial hemodynamic assessment. If prolonged monitoring is indicated, especially in patients who are not ventilated and sedated, the EDM should be replaced with PAC. ${ }^{[17]}$ In this capacity, the EDM can be used to help determine the presence and the nature of hemodynamic abnormality and provide time before image-guided placement of central venous lines can be performed or be used as a sole hemodynamic assessment modality if the patient can be successfully resuscitated within a set period of time. We set this suggested time interval around the $75^{\text {th }}-85^{\text {th }}$ percentile of EDM time usage in this study or approximately 6-8 $\mathrm{h}$. The rationale for this approach is that majority of patients in this series responded to the interventions directed by the EDM within that period of time and those who did not respond to such interventions usually required prolonged hemodynamic monitoring ( $>24$ hours). In addition, patient comfort level and sedation requirements may be lower when PAC is used for the long-term monitoring as opposed to EDM.

Several exceptions to this rule can be entertained. One of these exceptions would be in cases involving organ procurement, in which case the EDM may potentially be utilized up to and including the organ procurement procedure. In other cases, there may be a contraindication or inability to obtain central venous access. For example, the EDM can be used in a patient who is bacteremic/ fungemic and in whom one wishes to limit the number of invasive central venous monitoring devices. At times, patients who present with limited central venous access options (i.e., patients with end-stage renal disease and/or multiple prior venous access procedures) can be resuscitated for longer periods of time utilizing the EDM as the sole hemodynamic monitoring device.

Esophageal Doppler probe insertion can be performed nasally or orally. In this study, oral insertion was more likely in organ donors (86\%) than nonorgan donors (NOD) $(22 \%)$, depending mainly on patient tolerability factors and sedation requirements. Oral insertion in NOD 
patients was utilized in patients who were deeply sedated, already had pre-existing nasal catheters (nasogastric or postpyloric) or in cases where the EDM could not be placed nasally (due to anatomic size limitations) in a sedated patient without immediately available adequate central venous access catheter for PAC insertion.

Much like in the previous published reports, we tended to follow only selected variables when it comes to observational trends in the EDM data. ${ }^{[4,7]}$ In fact, even when the EDM did not seem to provide the correct absolute values of cardiac performance parameters, it did provide clinically useful hemodynamic parameter trends. ${ }^{[4,7-8]}$

At times, we utilized the EDM for a quick-look hemodynamic assessment, followed by a period of resuscitation, followed by re-insertion and another quick-look confirmation of patient hemodynamic status. In this manner, the EDM served as a confirmatory tool, which when combined with utilization of resuscitation end-points, offered a more complete hemodynamic and clinical picture of the patient status. With the use of topical local anesthetic agent, this clinical maneuver can be easily performed even in a non-sedated, nonintubated patient.

Confirmatory laboratory parameters used to verify and reaffirm EDM observations in this study were lactic acid level and base excess. Both of these parameters showed significant improvement as a result of EDMguided resuscitation, as evidenced by decreased mean post-resuscitation lactic acid levels and decreased mean post-resuscitation base deficit. De la Torre, et al., also advocate the use of resuscitation endpoints in conjunction with the EDM. ${ }^{[18]}$

In cases where the PAC and the EDM were in place simultaneously, analogous data were obtained (cardiac output) from the respective modalities. Previous reports show that the correlation between cardiac output measurements obtained by the EDM and the PAC tends to be very good, with the reported range of correlation coefficient between 0.52 and 0.98 and the mean correlation coefficient of 0.89 from collected literature reviews. ${ }^{[14,16]}$ However, these studies lack significantly in their methodologies and do not convincingly demonstrate the interchangeability of the two methods. When examined closely, our data shows that the EDM tends to underestimate $\mathrm{CO}$ in the lower range of measurements and tends to overestimate $\mathrm{CO}$ in the upper range of measurements [Figure 1]. One previous study also reported that when compared to the PAC, the EDM tended to consistently underestimate cardiac output in women with preeclampsia. ${ }^{[8]}$ Based on these findings, caution has to be exercised when EDM and the PAC are being used interchangeably and although measurements obtained by the two methods are generally in agreement, clinicians should be advised that perhaps a more reliable method of transitioning between the two techniques should be based on establishing and defining new measurement trends following change in measuring method. Keeping with previously published observations, it has to be noted that as with most monitoring equipment utilized in resuscitation of critically ill patients, both the EDM and the PAC have to be used in the context of the overall clinical picture and can only constitute an adjunct to good clinical judgment. ${ }^{[8,19-20]}$

We also noted a statistically significant correlation between the absolute change in left ventricular ejection time (LVET) and the change in base excess. Although the complete significance of this finding is unknown, it may point to the LVET as a resuscitative monitoring tool. Of interest, the change in lactic acid level did not seem to correlate with the change in base excess or the change in LVET. It is uncertain why one, but not the other resuscitation end-point would correlate with an EDM parameter. Perhaps other, clinical factors such as amount and rate of administration of resuscitative fluids or presence of organ dysfunction, contributed to this observation.

The EDM can provide the clinician with other potential advantages over the PAC. First, the EDM takes approximately 10 min less to insert than the PAC and is less expensive than the PAC. ${ }^{[4]}$ The cost of placing the PAC is between $\$ 80$ and $\$ 100$ greater than the cost of EDM placement for each insertion. ${ }^{[4]}$ Second, it can be used continuously or discontinuously and does not require central venous access to initiate patient monitoring. In several patients in this series, the EDM obviated the need for central venous access and only peripheral intravenous access was used. Thus, clinicians have an option for accurate hemodynamic monitoring in settings where it may be logistically difficult to obtain this data - the 


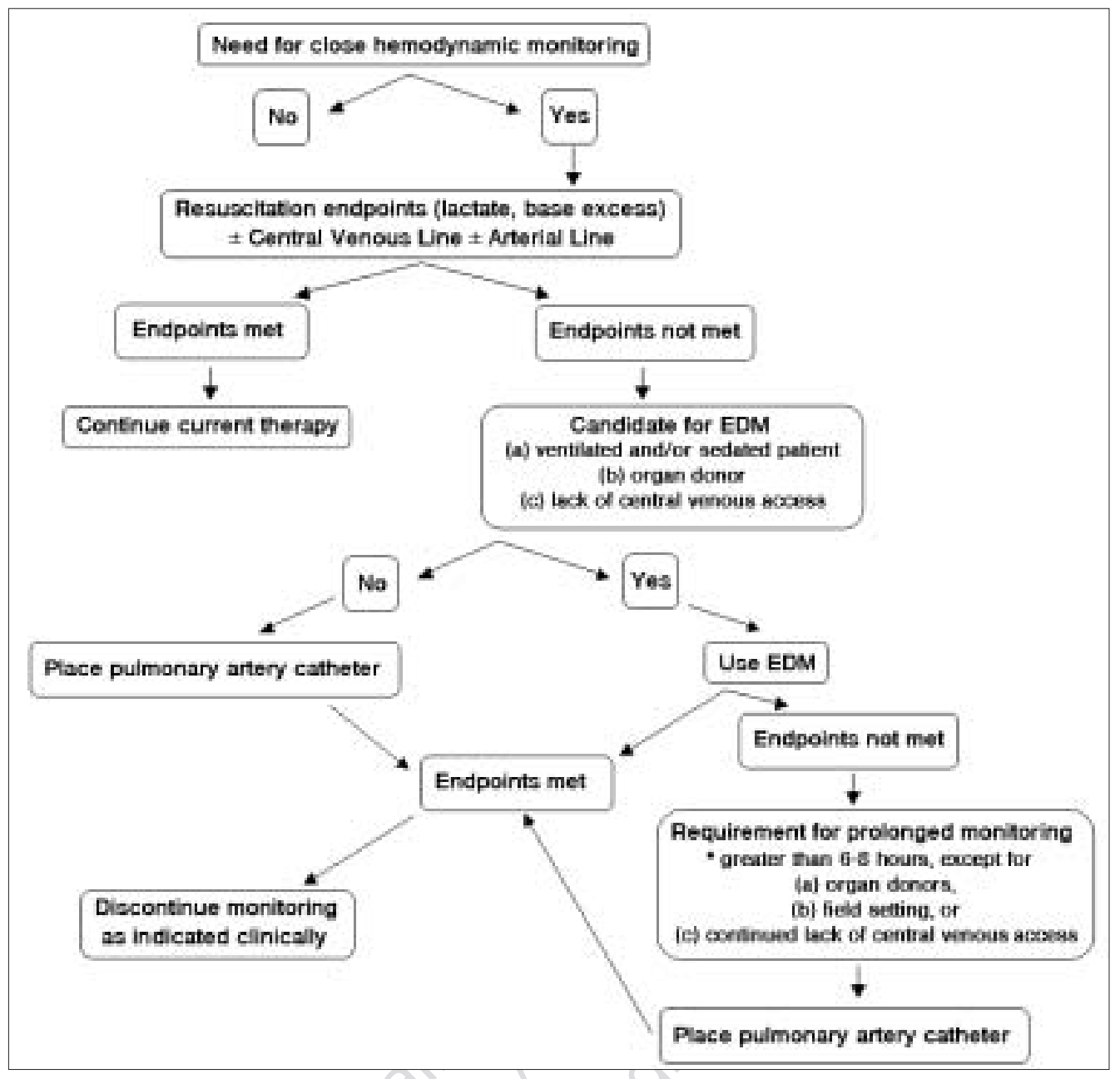

Figure 2: Algorithm for esophageal Doppler monitoring in the ICU. PAC = Pulmonary artery catheter, EDM = Esophageal Doppler monitor, * In these clinical situations, the EDM could be utilized for much longer periods of time (in this study, up to $19 \mathrm{~h}$ )

emergency department or the general hospital ward. In fact, the ease of EDM deployment and signal acquisition make it possible for trained allied health professionals to place, use and communicate the EDM data to a central location, an exciting option for organ procurement teams, the military, rescue and pre-hospital personnel, as well as disaster response medical teams. ${ }^{[20]}$ A proposed algorithm for EDM use in the ICU is presented [Figure 2].

\section{Conclusions}

The EDM offers an alternative method of hemodynamic assessment in critically ill patients, with a broad spectrum of potential indications. With some exceptions, we advocate its use in ventilated and/or sedated patients for episodic care requiring short- to moderate-term (6 to $8 \mathrm{~h}$ ) hemodynamic monitoring. For patients who require long-term hemodynamic monitoring or patients who are not sedated, the PAC remains the best option. When compared to PAC, the EDM tends to underestimate $\mathrm{CO}$ in the lower range and overestimate $\mathrm{CO}$ in the upper range of measurements. We recommend EDM use concurrently with end-points of resuscitation. A proposed algorithm for EDM use in the ICU is presented.

\section{References}

1. Marik PE. Pulmonary artery catheterization and the esophageal Doppler monitoring in the ICU. Chest 1999;116:1085-91.

2. Connors AF Jr, Speroff T, Dawson NV, Thomas C, Harrell FE, Wagner $D$, et al. The effectiveness of right heart catheterization in the initial care of critically ill patients. JAMA 1996;276:889-97.

3. Shoemaker WC, Belzberg H, Wo CC, Milzman DP, Pasquale MD, Baga $L$, et al. Multicenter study of noninvasive monitoring system as alternatives to invasive monitoring of acutely ill emergency patients. Chest 1998;114:1643-52.

4. Stawicki SP, Hoff WS, Cipolla J, deQuevedo R. Use of non-invasive esophageal echo-Doppler system in the ICU: A practical 
experience. J Trauma 2005;59:506-7.

5. Dark PM, Singer M. The validity of trans-esophageal Doppler ultrasonography as a measure of cardiac output in critically ill adults. Intensive Care Med 2004;30:2060-6.

6. Seoudi HM, Perka MF, Hanrahan A, Angood PB. The esophageal Doppler monitor in mechanically ventilated surgical patients: Does it work? J Trauma 2003;55:720-6.

7. Kim K, Kwok I, Chang H, Han T. Comparison of cardiac outputs of major burn patients undergoing extensive early escharectomy: Esophageal Doppler monitor versus thermodilution pulmonary artery catheter. J Trauma 2004;57:1013-7.

8. Penny JA, Anthony J, Shennan AH, De Swiet M, Singer M. A comparison of hemodynamic data derived by a pulmonary artery catheter and the esophageal Doppler monitor in preeclampsia. Am J Obstet Gynecol 2000;183:658-61.

9. Traub D, Hoey BA, Stawicki SP, Hoff WS, Anderson HL, Wright S, et al. Novel indications for the use of a non-invasive esophageal echoDoppler system in the ICU. Crit Care Med Suppl 2002;30:A63.

10. Ueda M, Yokota S, Nakata F, Kaseno S, Sakuraya N, Kemmotsu O. Clinical evaluation of esophageal Doppler cardiac output measurement during general anesthesia. J Anesth 1989;3:178-82.

11. Bernardin G, Tiger F, Fouche R, Mattei M. Continuous noninvasive measurement of aortic blood flow in critically ill patients with a new esophageal echo-Doppler system. J Crit Care 1998;13:177-83.

12. Singer M. Esophageal Doppler monitoring of aortic blood flow: Beat by beat cardiac output monitoring. Int Anesthesiol Clin 1993;31:99125.

13. Reiter A, Mauritz W, Jordan B, Lang T, Polzl A, Pelinka L, et al. Improving risk adjustment in critically ill trauma patients: The
TRISS-SAPS Score. J Trauma 2004;57:375-80.

14. Laupland KB, Bands CJ. Utility of esophageal Doppler as a minimally invasive hemodynamic monitor: A review. Can J Anesth 2002;49:393-401.

15. Valtier B, Cholley BP, Belot JP, de la Coussaye JE, Mateo J, Payen DM. Noninvasive monitoring of cardiac output in critically ill patients using transesophageal Doppler. Am J Respir Crit Care Med 1998;158:77-83.

16. Grindlinger G, Ahmad S, Desjardins S. Use of the esophageal Doppler and sublingual capnometer in the hemodynamic assessment of mechanically ventilated surgical patients. Chest Suppl 2004;126:874S.

17. Stawicki SP, Cipolla J, McQuay N, Hoff WS, Hoey BA, Grossman MD. Move over, Mr Swan-Ganz: Finding a place for the esophageal doppler monitoring system in the ICU. Crit Care Med Suppl 2005;33:A59.

18. de la Torre AN, Fisher A, Wilson DJ, Reitsma W, Goerlitz F, Koneru B. Minimally invasive optimization of organ donor resuscitation: Case reports. Prog Transplant 2005;15:27-32.

19. Han T. The esophageal Doppler monitor is useful for fluid management during the perioperative and intensive care periods. $J$ Trauma 2005;59:508.

20. Cipolla J, Stawicki S, Spatz D. Hemodynamic monitoring of organ donors: A novel use of the esophageal echo-Doppler probe. Am Surg 2006;72:500-4.

Source of Support: Nil, Conflict of Interest: None declared

\section{Author Help: Online Submission of the Manuscripts}

Articles can be submitted online from http://www.journalonweb.com. For online submission articles should be prepared in two files (first page file and article file). Images should be submitted separately.

1) First Page File:

Prepare the title page, covering letter, acknowledgement, etc., using a word processor program. All information which can reveal your identity should be here. Use text/rtf/doc/pdf files. Do not zip the files.

2) Article file:

The main text of the article, beginning from Abstract till References (including tables) should be in this file. Do not include any information (such as acknowledgement, your names in page headers, etc.) in this file. Use text/rtf/doc/pdf files. Do not zip the files. Limit the file size to $400 \mathrm{~kb}$. Do not incorporate images in the file. If file size is large, graphs can be submitted as images separately without incorporating them in the article file to reduce the size of the file.

3) Images:

Submit good quality colour images. Each image should be less than $\mathbf{4 0 0} \mathbf{~ k b}$ in size. Size of the image can be reduced by decreasing the actual height and width of the images (keep up to about 4 inches) or by reducing the quality of image. All image formats (jpeg, tiff, gif, bmp, png, eps, etc.) are acceptable; jpeg is most suitable. The image quality should be good enough to judge the scientific value of the image. Always retain a good quality, high resolution image for print purpose. This high resolution image should be sent to the editorial office at the time of sending a revised article.

4) Legends:

Legends for the figures/images should be included at the end of the article file. 